\title{
Accelerating sample preparation of graded thermoelectric materials using an automatic powder feeding system
}

\author{
Hua-Yan $\mathrm{Pu}^{1} \cdot$ Rong-Qing $\mathrm{Xie}^{1} \cdot$ Yan Peng $^{1} \cdot$ Yang Yang $^{1}\left({ }^{-} \cdot\right.$ Shi-Yang $\mathrm{He}^{2} \cdot$ \\ Jun $\mathrm{Luo}^{2,3} \cdot$ Yi Sun ${ }^{1} \cdot$ Shao-Rong Xie ${ }^{1} \cdot J_{u n}$ Luo $^{1}$
}

Received: 30 September 2018/Revised: 14 March 2019/Accepted: 4 July 2019/Published online: 26 August 2019

(C) The Author(s) 2019

\begin{abstract}
In recent years, the development of techniques for the controlled preparation of functional graded materials (FGMs) has become a vigorous research field. In this study, to improve the efficiency and accuracy of sample preparations, an automated feeding system based on gravimetric principles for dry powder with three dosing feeders is designed and realized. The feeding rate and accuracy can be regulated by coordinating the protruded length $L(\mathrm{~mm})$ and rotational speed $V(\mathrm{r} / \mathrm{min})$ of the feeder stirrer. To demonstrate this automatic sample preparation system, the well-known thermoelectric material $\mathrm{Bi}_{x} \mathrm{Sb}_{2-x}$ $\mathrm{Te}_{3}(x=0.3,0.4,0.5,0.6,0.7$ and 0.8$)$ is selected and prepared by the developed system, and the composition distribution of the functional graded material is characterized. Experimental results show that the $\mathrm{Bi}_{x} \mathrm{Sb}_{2-x} \mathrm{Te}_{3}$ $(x=0.3-0.8)$ functionally graded material crystalizes in the rhombohedral phase after hot-pressing sintering and annealing and the prepared sample has a good gradient
\end{abstract}

Jun Luo listed first in author group is the corresponding author.

Electronic supplementary material The online version of this article (https://doi.org/10.1007/s40436-019-00269-y) contains supplementary material, which is available to authorized users.

Yang Yang

yangyang_shu@shu.edu.cn

$\triangle$ Jun Luo

junluo@shu.edu.cn

1 School of Mechatronic Engineering and Automation, Shanghai University, Shanghai 200444, People's Republic of China

2 School of Materials Science and Engineering, Shanghai University, Shanghai 200444, People's Republic of China

3 Materials Genome Institute, Shanghai University, Shanghai 200444, People's Republic of China composition distribution. This verifies the reliability and accuracy of the feeding system. The concept of samples with a gradient component and application of the automatic powder feeding system could considerably accelerate the research and development of new materials.

Keywords Powder feeding - Automatic system . Functional graded material . Thermoelectric

\section{Introduction}

Thermoelectricity is currently considered as a plausible means of producing "clean" electrical energy from virtually any type of waste heat [1]. The demand for higher thermoelectric device efficiency combined with mandatory cost reductions per watt of thermoelectrically produced power helps drive upstream material research. Functional graded materials (FGMs) have primarily been studied for fabricating thermoelectric modules for assembling and welding of $\mathrm{p}$ - and $\mathrm{n}$ - legs to enhance the thermoelectric energy conversion efficiency in thermoelectrics [2]. FGMs are a useful means of improving the efficiency and usable temperature range of thermoelectrics. An FGM can undergo a continuous change in composition with annealing and other processes. Therefore, it might be used as an ideal sample to screen out the sample composition with the best thermoelectric performance. Thus, an FGM with a quasi-continuous composition distribution satisfies the sample requirement of the emerging materials genome initiative (MGI) and could lead to further research and improvements in thermoelectric conversion efficiency.

In actual operation, the precision of weighing a highpurity material powder has a great influence on the outcome of the preparation. The greatest challenge in the 
process is that the operator must keep dosing the powder without scattering small quantities of powders into a small container to ensure weighing accuracy. In addition, the aerosol formation may pose a risk of cross-contamination because of the repeated opening of the storage container for the powder. In most laboratories, a spatula is usually used to weigh accurately and dispense powders by hand, which is an imprecise, time-consuming, tedious, and even highrisk (e.g., samples may be poisonous and harmful) manual sample preparation method. Therefore, introducing a precise sample feeding system to accelerate the development of thermoelectric materials is necessary.

Similar to the requirements for sample preparation in the field of thermoelectrics, handling small quantities of powder is required in a variety of industries and processes, such as in the preparation of pharmaceutical products, the continuous preparation of paints, inks, or glazes, and the formation of a two-dimensional image or a succession of two-dimensional images [3-5]. Dry powders are primarily defined by the fact that the adhesive and cohesive forces outweigh gravity, and the particle sizes of processed powders become increasingly smaller in biotechnology, pharmaceuticals, and materials, which leads to mostly unwanted agglomeration (clumping) and surface adhesion. Thus, using conventional conveyor systems for dispensing is more difficult. Yang and Evans [3] have provided an overview of various micro-feeders and low-dose dosing systems and their working principles. The methods for metering and dosing powders, including pneumatic, volumetric, screw/auger, electrostatic, and vibratory methods, are nearly all based on volumetric or gravimetric principles.

Most dosing techniques are based on volumetric principles. An aspirating-dispensing head for volumetric dosing can draw off powder in discrete metered quantities of 0.5-10 mg [6]. However, this pneumatic method cannot provide continuous automatic metering and powder dispensing. Volumetric metering can be achieved using continuous aspiration from a grooved conveyor [7]. The machine can be used to dispense powder with high instantaneous precision (less than $1 \%$ variation) and at high speeds $(3-11 \mathrm{~g} / \mathrm{s})$. A volumetric micro-feeder based on a cylinder piston system (i.e., a powder pump) was presented by Besenhard et al. [8], and this feeder allowed for accurate micro-feeding and feed rates of a few grams per hour, even for very fine powders. However, volumetric dosing generates errors as a result of particle packing, the potential effects of humidity, electrostatic effects, and batch-to-batch variations in the powder. Small differences in particle size distribution between different powder batches can disproportionately affect the particle packing efficiency and some waste powder is always created [9].
Gravimetric techniques are better suited for providing accurate low (or micro) feeding. For example, microfeeding has successfully been performed using vibrating capillaries or rods [10-12], an ultrasonic regime [13-15], auger methods [16], vibratory channels [17, 18], and vibrating spatulas [19]. Compared to volumetric powder feeders, a system based on gravimetric feeding principles can not only guarantee precise and effective feeding but also can have outstanding robustness. However, conventional weighing units are often too slow for small doses (below $200 \mathrm{mg}$ ) [3].

In this study, an automatic feeding system based on gravimetric principles is developed to feed powders quickly, continuously, reliably, and accurately. A successful application involving preparation of samples with a gradient component for FGMs is presented. Bismuth telluride $\left(\mathrm{Bi}_{x-}\right.$ $\mathrm{Sb}_{2-x} \mathrm{Te}_{3}$ ) compounds and alloys are the best materials for room-temperature dominated industrial applications because the materials have a layered crystal structure with a narrow electronic band gap [20]. Therefore, $\mathrm{Bi}_{x} \mathrm{Sb}_{2-x} \mathrm{Te}_{3}$ ( $x=0.3,0.4,0.5,0.6,0.7$ and 0.8$)$ compounds are used to study the composition distribution of FGMs. Using the automatic feeding system, we demonstrated that $\mathrm{Bi}_{x} \mathrm{Sb}_{2-x}$ $\mathrm{Te}_{3}$ FGMs formed a single phase following hot-pressing sintering. We analyzed its composition to prove that the prepared samples had a gradient component, thus verifying the feasibility of the system.

\section{Materials and methods}

\subsection{Materials}

Homogeneous p-type $\mathrm{Bi}_{x} \mathrm{Sb}_{2-x} \mathrm{Te}_{3}$ samples with $x$ values of $0.3,0.4,0.5,0.6,0.7$ and 0.8 were prepared. As shown in Fig. 1, a compositional gradient sample must be formed in

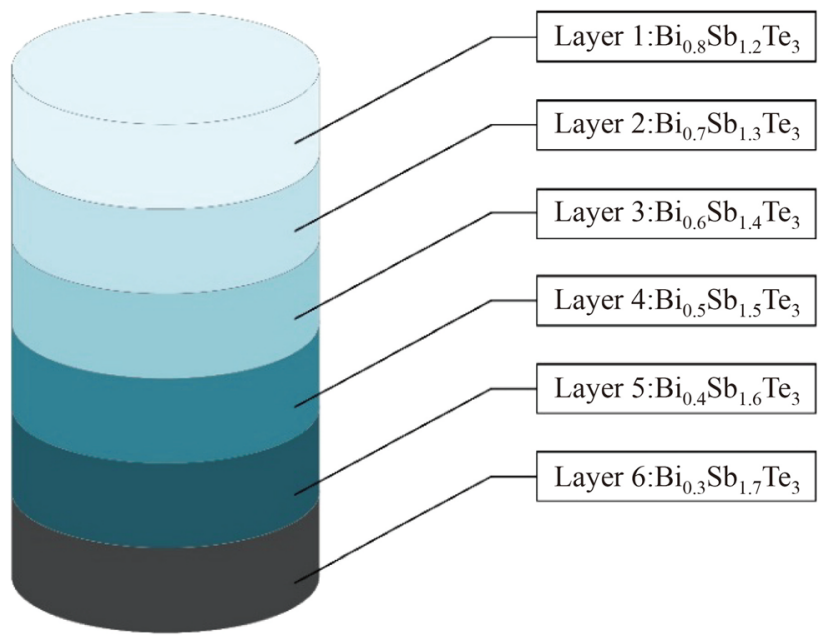

Fig. 1 Schematic of the FGM sample in this work 
Table 1 Properties of sample powders

\begin{tabular}{lllll}
\hline Material & Supplier & Purity/\% & Mesh number & $\begin{array}{l}\text { Bulk density/ } \\
\left(\mathrm{g} \cdot \mathrm{cm}^{-3}\right)\end{array}$ \\
\hline $\mathrm{Bi}$ & Aladdin industrial corporation & 99.99 & 200 & 9.8 \\
$\mathrm{Sb}$ & Aladdin industrial corporation & 99.99 & 200 & 6.7 \\
$\mathrm{Te}$ & Aladdin industrial corporation & 99.99 & 100 & 6.3 \\
\hline
\end{tabular}

Table 2 Sample formulas (unit: $\mathrm{mg}$ )

\begin{tabular}{|c|c|c|c|c|c|c|}
\hline \multirow[t]{2}{*}{ Element } & \multicolumn{6}{|c|}{ Layer } \\
\hline & 1 & 2 & 3 & 4 & 5 & 6 \\
\hline $\mathrm{Bi}$ & 314 & 209 & 209 & 209 & 139 & 105 \\
\hline $\mathrm{Sb}$ & 275 & 226 & 284 & 365 & 325 & 345 \\
\hline $\mathrm{Te}$ & 719 & 547 & 638 & 765.6 & 638 & 638 \\
\hline
\end{tabular}

six layers. Each layer is prepared using three types of fine powders $(\mathrm{Bi}, \mathrm{Sb}$, and $\mathrm{Te}$ ) in various ratios. The properties of the powders, including their purity and mesh number, are listed in Table 1. The sample components show a gradient change in the longitudinal direction, and the content of each layer is given in Table 2.

To improve the efficiency and accuracy over those of manual operation, a feeding system is developed to prepare samples with a gradient component. A complete sample preparation procedure as shown in Fig. 2 is described as follows:

(i) Use the developed system and stack the raw materials into gradient compositions;

(ii) Densify the FGMs by hot-pressing, and then anneal for homogenization;

(iii) Cut along the axial direction and polish into a thin slab with a thickness of $1.5 \mathrm{~mm}$ for performance characterization.

\subsection{Equipment and process}

\subsubsection{Automatic powder feeding system}

The developed automatic system for stacking raw materials into gradient compositions is shown in Fig. 3 and consists of the following three modules.

(i) Feeding module. As shown in Fig. 3b, the module contains horizontal and vertical driving units, a rotating unit, and three dosing feeders. These two linear driving units are based on two motorized stages, respectively. Three dosing feeders (Mettler Toledo, QH012-ZNMW 11150115 , Switzerland) are attached to the platform of the horizontal driving unit. Each dosing feeder contains one

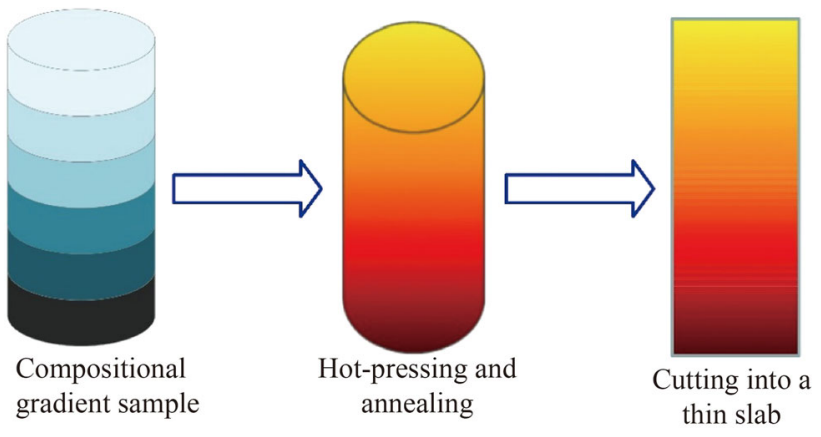

Fig. 2 Schematic procedure for preparing an FGM sample

type of powder, and different dosing feeders could be switched by the horizontal driving unit to achieve sequential feeding for different powders. The rotating unit is composed of a DC brushed servo motor and a driving shaft. The driving shaft is mounted on the platform of the vertical unit and can be rotated by the DC motor. The structure of the dosing feeder is shown in Fig. 3c. A thin and long groove was fabricated at the stirrer of the feeder. After the feeder containing the required powder is moved under the shaft by the horizontal unit, the stirrer rotates and slides down as driven by the vertical and rotating units, and then the powder can outflow from a glass container through the groove into a small reservoir on the balance. The feeding rate and accuracy can be regulated by coordinating the rotational speed $V(\mathrm{r} / \mathrm{min})$ and the protruded length $L(\mathrm{~mm})$ of the stirrer through the shaft. After dosing is completed, the end of the stirrer can seal the dosing feeder when it returns to its initial position.

(ii) Weighing module. A micro precision electronic balance (Advance Mass Verification Balance, Mettler Toledo XSE105DU, Switzerland) is placed under the dosing feeders and integrated with the dosing unit to form a closed-loop control system, as shown in Fig. 3a. The balance records the actual weight value and sends the feedback signal to the controller in real-time to control the feeding rate and improve the feeding accuracy of the system. The balance has a readability of $0.01 \mathrm{mg}$ and a weighing range $0-41 \mathrm{~g}$, which can meet the requirements of high-precision and large-range dosing.

(iii) Transmission module. The transmission module is developed to transport the reservoirs before and after dosing to improve the degree of automation. The module 


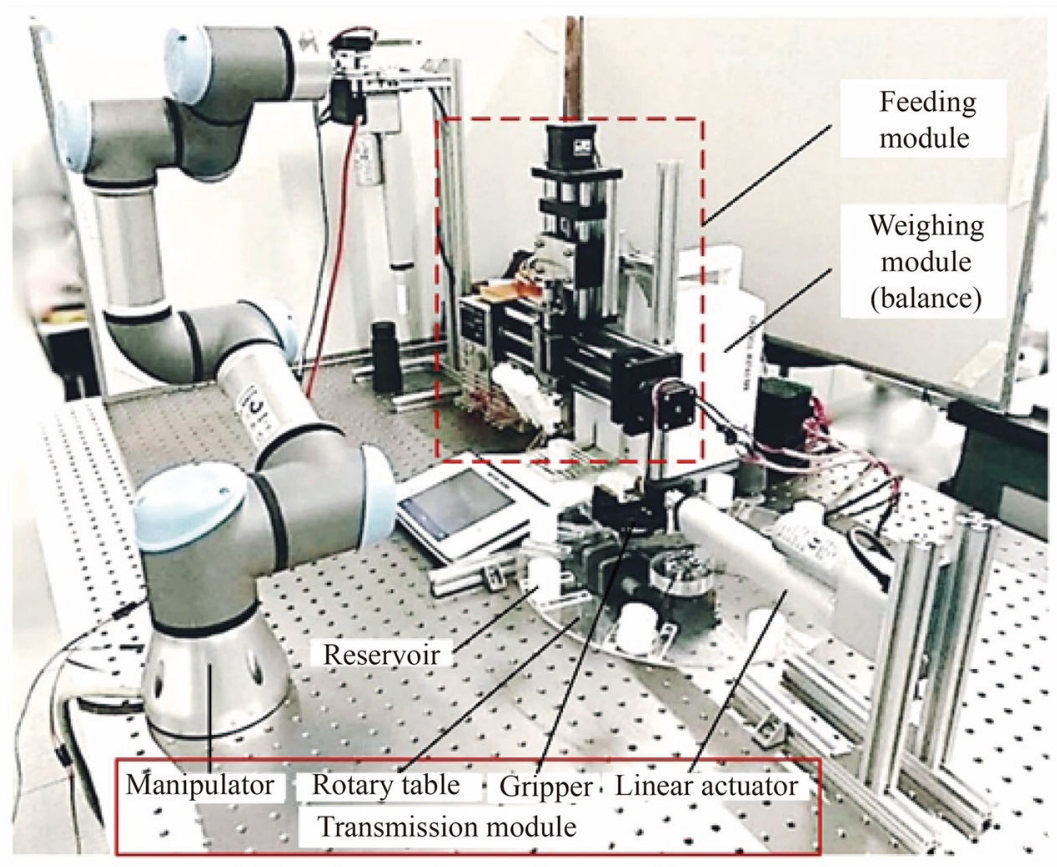

(a)

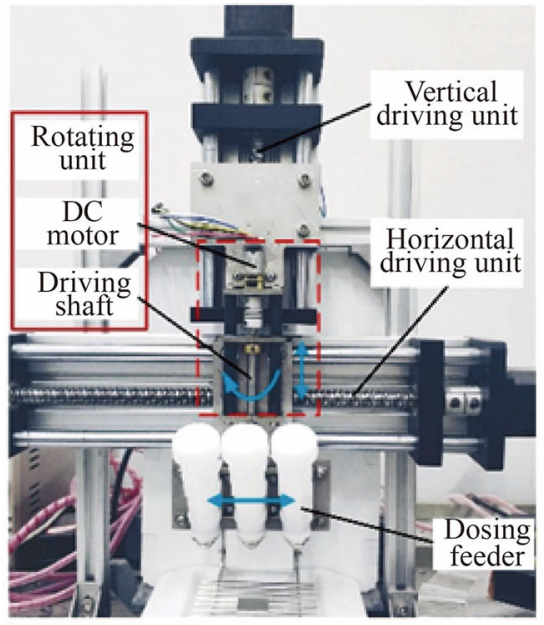

(b)

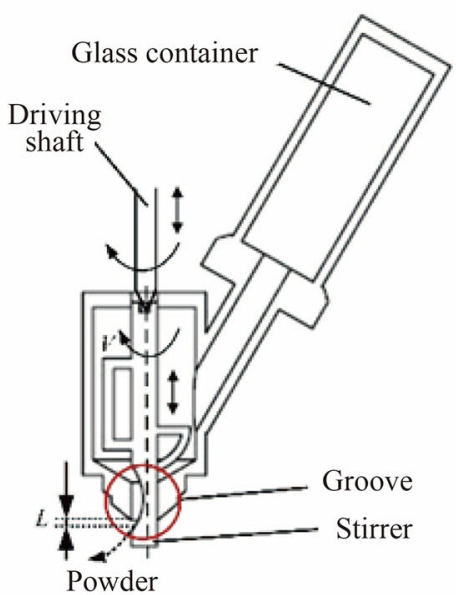

(c)

Fig. 3 Automated powder feeding system a system setup $\mathbf{b}$ feeding module $\mathbf{c}$ schematic of the dosing feeder

contains a charging unit and 6-DoF manipulator (Universal Robots, UR3, Denmark), as shown in Fig. 3a. To eliminate the risk of cross-contamination, using a single reservoir for each mixture ratio of the powder is necessary. The charging unit conveys the reservoirs to the balance table in sequence before dosing. It contains a linear actuator, gripper, and rotary table. Once the reservoir is moved to the desired position by the rotary table, the gripper carries and conveys the reservoir to the balance table via the linear actuator. After the dosing is completed, the reservoir is transported by the 6-DoF manipulator to the desired location to wait for post-processing operations such as compaction, forming, and sintering.

\subsubsection{Operation space analysis}

The feeding rate and accuracy of the system can be regulated by coordinating the rotational speed $V(\mathrm{r} / \mathrm{min})$ and the protruded length $L(\mathrm{~mm})$ of the stirrer of the dosing feeder. As shown in Fig. 3c, a greater protruded length exposes a larger groove in the stirrer, and more powder outflows. In addition, the stirrer stirs the powder to overcome adhesion and promote flowability. Thus, a higher rotational speed of the stirrer can improve the flowability of the powder, thereby accelerating the feeding speed. Previous experiments have shown that the protruded length has a more obvious effect than does the rotational speed on the feeding 
rate. However, the responding speed of the system when regulating the rotational speed is faster than that when regulating the protruded length. Therefore, the rotational speed of the stirrer can be used for auxiliary and fine tuning to guarantee the accuracy, and enlarging the protruded length can significantly improve the feeding efficiency of the system. To determine the effect of the rotational speed, an experiment of feeding Bi powder was performed. The experimental setup was examined for various rotational speeds and $4 \mathrm{~mm}$ protruded lengths. The values of $V$ were varied from $20 \mathrm{r} / \mathrm{min}$ to $230 \mathrm{r} / \mathrm{min}$ at intervals of $35 \mathrm{r} / \mathrm{min}$. As shown in Fig. 4, higher rotational speed of the stirrer improved the flowability of powder and accelerated the feeding speed. The feeding mass linearly increased with the feeding time, which demonstrated the robust performance of the system. In Fig. 4, the feeding rate $f_{\mathrm{r}}(\mathrm{mg} / \mathrm{s})$ can be determined by the slope of the feeding characteristic curve according to Eq. (1). For different combinations of $V$ and $L$, it was found that the maximum and minimum feeding rates were obtained when $L=8 \mathrm{~mm}, V=230 \mathrm{r} / \mathrm{min}$ and $L=1 \mathrm{~mm}, V=20 \mathrm{r} / \mathrm{min}$, respectively. It was determined that the maximum and minimum feeding rates were $15.27 \mathrm{mg} / \mathrm{s}$ and approximately $0.19 \mathrm{mg} / \mathrm{s}$, respectively, by fitting the linear polynomial. A large range of the feeding rate represents a major advantage of the device, which enables the system to achieve both micro- and fast feeding by regulating the feeding rate.

$f_{\mathrm{r}}=\frac{\Delta m}{\Delta t}$.

\subsubsection{Feeding strategy}

This study proposes a control strategy for improving the feeding speed and accuracy. Larger values of protruded length and rotational speed were adopted first and were then gradually reduced to guarantee the feeding accuracy after the feeding mass approached the desired value. A

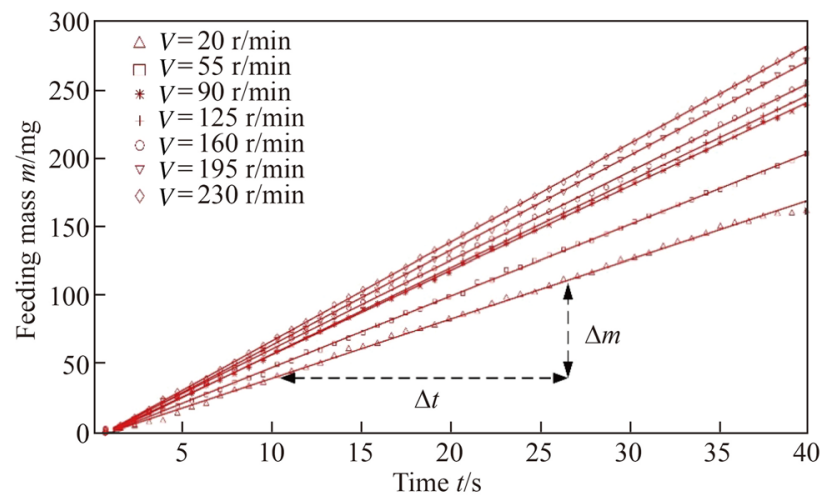

Fig. 4 Feeding characteristics curve $(V=20-230 \mathrm{r} / \mathrm{min}, L=4 \mathrm{~mm})$ single feeding event can be divided into fast and precise feeding stages. When the difference between the target mass value $m_{\mathrm{t}}$ and the measured mass value $m_{\mathrm{b}}$ feedback by the balance is more than $\varepsilon_{1}$, the fast feeding stage starts with a large $L$ and $V$ by open loop control. The precise feeding stage will start once the difference of $m_{\mathrm{t}}$ and $m_{\mathrm{b}}$ is between $\varepsilon_{2}$ and $\varepsilon_{1}$, and these two feeder parameters will be reduced to smaller values to ensure the feeding accuracy. A real-time proportion integration differentiation (PID) algorithm with position regulation was used in this stage. Finally, the feeding event will be finished when the difference is less than $\varepsilon_{2}$.

$\left\{\begin{array}{l}m_{\mathrm{t}}-m_{\mathrm{b}}>\varepsilon_{1}, \\ \varepsilon_{1} \geqslant m_{\mathrm{t}}-m_{\mathrm{b}}>\varepsilon_{2}, \\ m_{\mathrm{t}}-m_{\mathrm{b}}<\varepsilon_{2} .\end{array}\right.$

As shown in Fig. 5, the system is controlled by a programmable logic controller (PLC, Mitsubishi, FX3U32MR/DS, Japan), which has $32 \mathrm{I} / \mathrm{O}$ ports, of which 15 input and 15 output ports are used in this system. A PC running a Windows 10 operating system sends the desired feeding value to the PLC and receives the signals from the sensors to record the state of the system through a USB. According to the real-time weight feedback signal received from the balance through a RS232 bus, the PLC calculates the error between the target and measured mass values, and then sends the commands to the feeding system.

In the precise feeding stage, the flowing PID algorithms are used to improve the feeding accuracy and prevent overshoot of the system.

$U=K_{\mathrm{P}} e(t)+K_{\mathrm{I}} \int e(t) \mathrm{d} t+K_{\mathrm{D}} \frac{\mathrm{d} e(t)}{\mathrm{d} t}$,

where $U$ is control torque, $K_{\mathrm{P}}$ the proportional gain, $K_{\mathrm{I}}$ the integral gain, $K_{\mathrm{D}}$ the derivative gain. In our study, the values of the PID parameters were $K_{\mathrm{P}}=0.6, K_{\mathrm{I}}=0.4$, and $K_{\mathrm{D}}=1.5$.

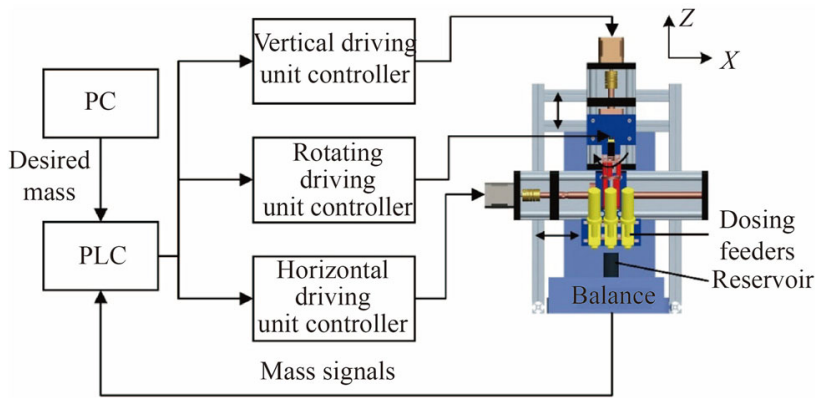

Fig. 5 Electrical system depicting the closed-loop control feeding system 


\subsubsection{Operating procedure}

The feeding procedure is described as follows.

Step 1 Fill sufficient powder in the glass containers of the feeders before feeding.

Step 2 Input the number of layers and weights of the components for each layer.

Step 3 Prepare one layer of the sample. One reservoir is conveyed from the rotary table to the balance by the charging unit, and then the three feeders feed the three types of powder in sequence by the feeding module. The reservoir is finally transported to the designated place by the manipulator.

Step 4 Repeat Step 3 to complete the preparation of all layers.

It should be noted that Steps 3 and 4 are automatically implemented by the system without any human intervention.

\subsection{Synthesis and characterization}

The prepared elemental layer mixture was first hot pressed in a $\Phi 10 \mathrm{~mm}$ graphite die at $673 \mathrm{~K}$ for $20 \mathrm{~h}$ under a uniaxial pressure of $75 \mathrm{MPa}$. Then, the hot-pressed cylindrical sample (compositional gradient ingot) was cut into small slices along the axial direction. Another part was sealed into a quartz tube using a Partulab device (MRVS-1003) at $10^{-3} \mathrm{~Pa}$ and annealed at $673 \mathrm{~K}$ for $24 \mathrm{~h}$. Finally, the samples were cut into a thin slab with a wire saw.

Phase identification of the sample was performed on a Panalytic Aeris diffractometer with $\mathrm{Cu}-\mathrm{K}_{\alpha}$ radiation $(40 \mathrm{kV} \times 15 \mathrm{~mA})$. The microstructure and sample composition were analyzed using a Zeiss GeminiSEM 300 scanning electron microscope equipped with an energy dispersive X-ray spectroscopy (EDXS) detector (Oxford Instruments).

\section{Results and discussion}

\subsection{Performance of the developed system}

The feeding process for the Bi powder in Layer 2 was used as an example to demonstrate the feeding performance of the developed system. As shown in Fig. 6, the trend in the feeding mass could be divided into fast and precise feeding stages in less than $3 \mathrm{~min}$. In the fast feeding stage, the protruded length $L(\mathrm{~mm})$ and rotational speed $V(\mathrm{r} / \mathrm{min})$ of the stirrer increased from 0 to $7.2 \mathrm{~mm}$ and from 0 to 160 $\mathrm{r} / \mathrm{min}$, respectively and were then held at the higher values. As a result, the feeding mass continuously increased with the high feeding rate through this stage. Once the feeding

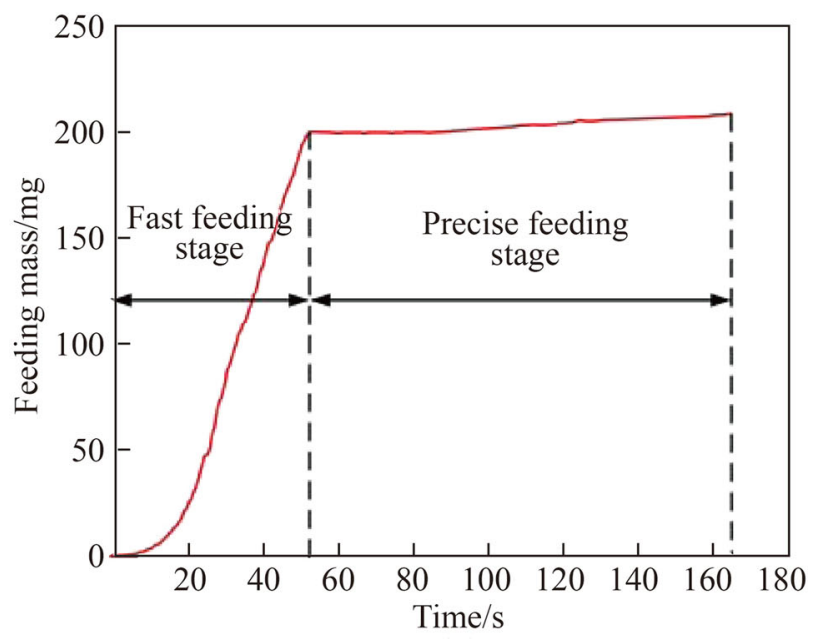

(a)

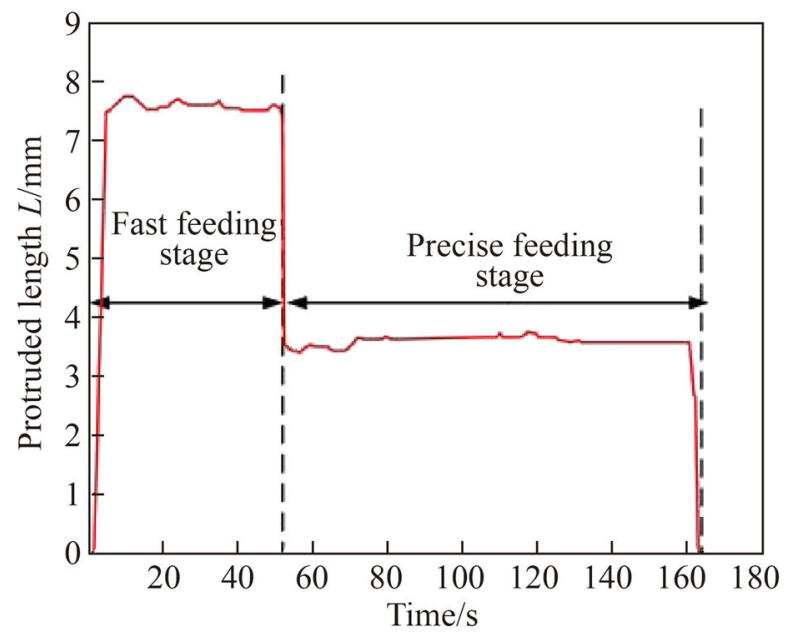

(b)

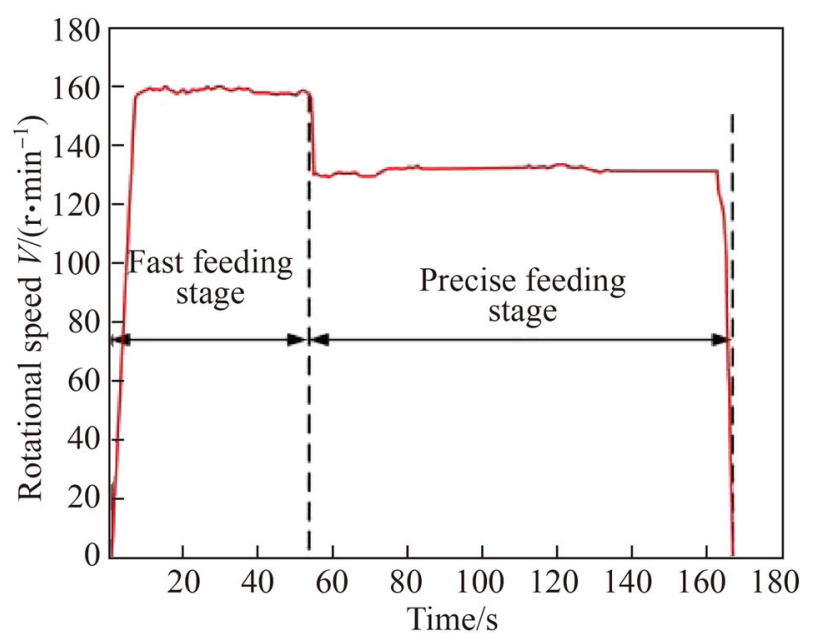

(c)

Fig. 6 Feeding process of Bi powder for preparing an FGM sample $\mathbf{a}$ feeding mass $\mathbf{b}$ protruded length $\mathbf{c}$ rotational speed

mass approached the desired value, the protruded length and rotational speed of the stirrer decreased to $6 \mathrm{~mm}$ and 
$130 \mathrm{r} / \mathrm{min}$, respectively. The feeding rate of the powder gradually slowed to prevent an overshoot and ensure accuracy in the precise feeding stage. By coordinating the rotational speed and protruded length of the stirrer, we improve the feeding efficiency using coarse feeding in the fast feeding stage, and the feeding accuracy could be guaranteed by fine feeding in the precise feeding stage.

To demonstrate repeatability, three samples were prepared by this system. The feeding errors of the automated system generated during the preparation of Sample 1 are listed in Table 3, and another two samples can be found in Table S1 and S2 of the Supplementary materials. It was confirmed that the feeding errors of all the components were less than $0.1 \mathrm{mg}$, and an acceptable accuracy was achieved by the developed system. In addition, to demonstrate the efficiency of the system, the preparation time for one sample prepared by the automated system was recorded and compared with the time for manual operation by a skilled operator. As shown in Table 4, the time-cost savings was approximately $50 \%$ by the system. Therefore, the developed system greatly reduced the manual burden on the experimentalists and improved efficiency, indicating it could have great significance for the development of new materials and new drugs.

\subsection{Phase identification}

Figure 7 shows a schematic of the X-ray diffraction (XRD) of a thin slab and XRD patterns of a compositional gradient ingot $\left(\mathrm{Bi}_{x} \mathrm{Sb}_{2-x} \mathrm{Te}_{3}, x=0.3-0.8\right)$. The asterisks indicate the presence of the Sb secondary phase in the sample. XRD data were collected from the surface of the thin slab. All of the reflections in the diffraction patterns could be assigned to the expected $\mathrm{Bi}_{x} \mathrm{Sb}_{2-x} \mathrm{Te}_{3}$ structure. After hot-pressing, it was observed that the main peaks were consistent with the standard pattern of $\mathrm{Bi}_{0.4} \mathrm{Sb}_{1.6} \mathrm{Te}_{3}$ (PDF\#72-1836). However, the impurity $\mathrm{Sb}$ remained, as depicted by the asterisks in Fig. 7. This could be attributed to an incomplete reaction time during hot-pressing. After annealing, the $\mathrm{Sb}$ impurities disappeared inside the thin slab, and the
Table 4 Cost time (s) in dosing Bi powder by the automated system and a manual method

\begin{tabular}{llllllr}
\hline & \multicolumn{2}{l}{ Layer } & & & & \\
\cline { 2 - 6 } & 1 & 2 & 3 & 4 & 5 & 6 \\
\hline Manual & 350 & 310 & 345 & 360 & 322 & 308 \\
Automated & 182 & 165 & 160 & 162 & 104 & 95 \\
\hline
\end{tabular}

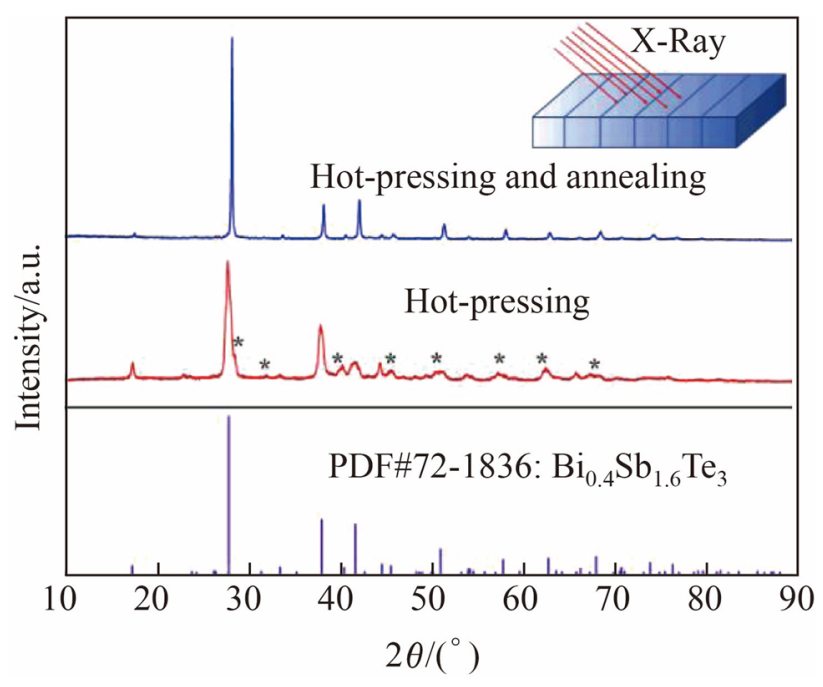

Fig. $7 \mathrm{X}$-ray diffraction patterns for a compositional gradient ingot of samples $\left(\mathrm{Bi}_{x} \mathrm{Sb}_{2-x} \mathrm{Te}_{3}, x=0.3-0.8\right)$ that have undergone a hotpressing process with annealing

phase composition was more in line with the phase of $\mathrm{Bi}_{x} \mathrm{Sb}_{2-x} \mathrm{Te}_{3}$.

\subsection{Microstructural characterization}

The middle area of the thin slab (Sample 1), which was annealed for $24 \mathrm{~h}$, was evaluated by SEM-EDXS analysis. The area that measured approximately $7.5 \mathrm{~mm}$ in length was divided into 14 small regions that were then used for EDS elemental mapping, as shown in Fig. 8b. The results for the SEM-EDX analysis of the small regions are shown

Table 3 Errors in automated dosing for preparing Sample 1

\begin{tabular}{|c|c|c|c|c|c|c|c|c|c|}
\hline \multirow[t]{2}{*}{ Layer } & \multicolumn{3}{|l|}{$\mathrm{Bi} / \mathrm{mg}$} & \multicolumn{3}{|l|}{$\mathrm{Sb} / \mathrm{mg}$} & \multicolumn{3}{|l|}{$\mathrm{Te} / \mathrm{mg}$} \\
\hline & Desired & Automated & Error & Desired & Automated & Error & Desired & Automated & Error \\
\hline 1 & 314.00 & 313.92 & 0.08 & 275.00 & 274.95 & 0.05 & 719.00 & 718.91 & 0.09 \\
\hline 2 & 209.00 & 208.95 & 0.05 & 226.00 & 226.01 & -0.01 & 547.00 & 546.96 & 0.04 \\
\hline 3 & 209.00 & 208.95 & 0.05 & 284.00 & 283.97 & 0.03 & 638.00 & 638.03 & -0.03 \\
\hline 4 & 209.00 & 209.03 & -0.03 & 365.00 & 364.92 & 0.08 & 765.60 & 765.52 & 0.08 \\
\hline 5 & 139.00 & 138.94 & 0.06 & 325.00 & 324.96 & 0.04 & 638.00 & 637.92 & 0.08 \\
\hline 6 & 105.00 & 104.91 & 0.09 & 345.00 & 344.99 & 0.01 & 638.00 & 637.95 & 0.05 \\
\hline
\end{tabular}




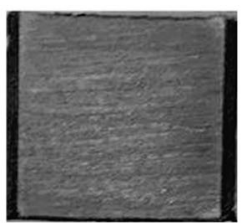

(a)

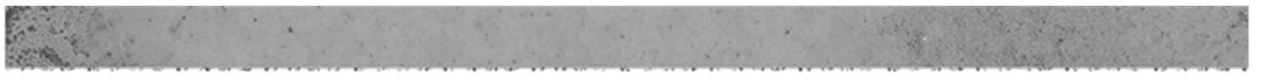

(b)

Fig. 8 Sample picture a thin slab picture b SEM joint picture

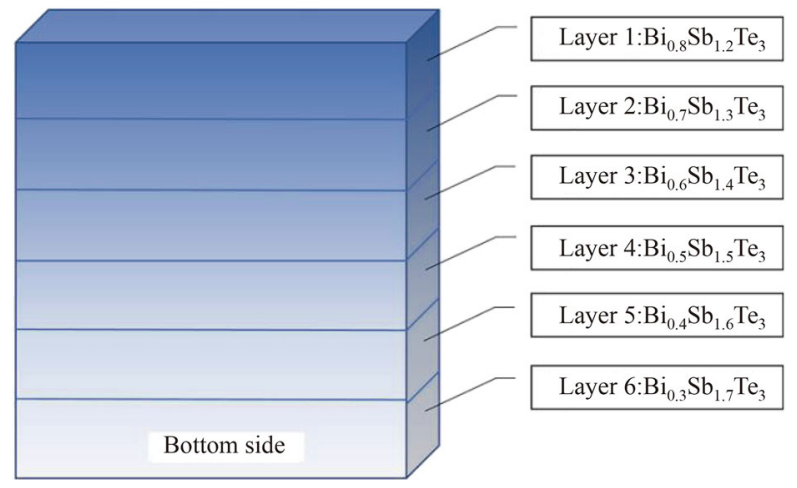

(a)

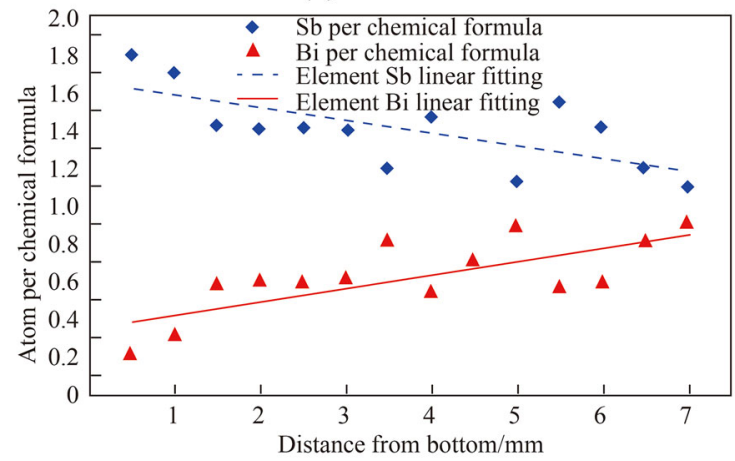

(b)

Fig. 9 Microstructural characterization a schematic of the thin slab as marked from the bottom side $\mathbf{b}$ element $\mathrm{Bi}$ and $\mathrm{Sb}$ content change trend along the slab from the bottom

in Fig. 9. The SEM joint picture of each area can be found in Fig. 8, and the details of the specific components are listed in Table S3 of the Supplementary materials (also see the composition distribution of Samples 2 and 3 in Tables S4 and S5, respectively, in the Supplementary materials.). It was found that the content of the element $\mathrm{Bi}$ increased from the bottom to the top side, and the thin slab showed a clear compositional gradient along the entire length of the sample. With verifying reproducibility, we repeated in triplicates, which showed a gradient distribution, as shown in the Supplementary materials for Samples 2 and 3. All prepared samples presented a gradient composition distribution when this dosing was used, which verified the reliability and accuracy of the feeding system.

\section{Conclusions}

In this study, an automated system based on gravimetric feedback was developed to prepare samples with a gradient component quickly, continuously, reliably, and accurately. The feeding rate and accuracy of the system could be regulated by coordinating the protruded length and rotational speed of the feeder stirrer, respectively. Using the automatic feeding system, we demonstrated that $\mathrm{Bi}_{x-}$ $\mathrm{Sb}_{2-x} \mathrm{Te}_{3}$ FGMs formed a single phase by hot-pressing sintering. The compositions were analyzed and the prepared samples were found to have a gradient component, thus verifying the feasibility of the system. The system significantly improved the feeding efficiency and accuracy compared to those of conventional manual operations. The proposed technology could be a low-cost solution for many applications in new materials and pharmaceutical development and production.

Acknowledgements This work was supported by the National Key Research and Development Program of China (Grant No. 2018YFB0703600), the National Natural Science Foundation of China (Grant Nos. 51772186, 91748116, 61773254, 61625304, and 61525305), in part by the Science and Technology Commission of the Shanghai Municipality (Grant Nos. 16DZ2260601 and 16441909400), in part by the Shanghai Rising-Star Program (Grant No. 17QA1401500), in part by the Shanghai Young Eastern Scholar Program (Grant Nos. QD2016029 and QD2016031), and in part by the Shanghai Sailing Program (Grant No. 17YF1406200).

Open Access This article is distributed under the terms of the Creative Commons Attribution 4.0 International License (http://crea tivecommons.org/licenses/by/4.0/), which permits unrestricted use, distribution, and reproduction in any medium, provided you give appropriate credit to the original author(s) and the source, provide a link to the Creative Commons license, and indicate if changes were made. 


\section{References}

1. Wu Y, Yang J, Chen S et al (2018) Thermo-element geometry optimization for high thermoelectric efficiency. Energy $147: 672-680$

2. Zhu T, Hu L, Zhao $X$ et al (2016) New insights into intrinsic point defects in $\mathrm{V}_{2} \mathrm{VI}_{3}$ thermoelectric materials. Adv Sci 3(7): 1600004

3. Yang S, Evans JRG (2007) Metering and dispensing of powder; the quest for new solid free forming techniques. Powder Technol 178(1):56-72

4. Norton FH (1952) Elements of ceramics. Addison-Wesley Press, Boston

5. Mott M, Evans JRG (1999) Zirconia/alumina functionally graded material made by ceramic ink jet printing. Mater Sci Eng A 271(12):344-352

6. Gupte AG, Kladders H, Struth H (1982) Device and process for drawing off very small quantities of powder. US Patent 4,350,049, 21 Sept 1982

7. Douche JP, Coulon JC, Bouttier P (1992) Device for metering pulverulent materials. US Patent 5,104,230, 14 Apr 1992

8. Besenhard MO, Fathollahi S, Siegmann E et al (2017) Microfeeding and dosing of powders via a small-scale powder-pump. Int J Pharm 519(1-2):314-322

9. Edwards D (2010) Applications of capsule dosing techniques for use in dry powder inhalers. Therapeutic Delivery 1(1):195-201

10. Chen X, Seyfang K, Steckel H (2012) Development of a micro dosing system for fine powder using a vibrating capillary. Part 1: the investigation of factors influencing on the dosing performance. Int J Pharm 433(1-2):34-41

11. Chen X, Seyfang K, Steckel H (2012) Development of a microdosing system for fine powder using a vibrating capillary. Part 2: the implementation of a process analytical technology tool in a closed-loop dosing system. Int J Pharm 433(1-2):42-50

12. Yang S, Evans JRG (2003) Computer control of powder flow for solid freeforming by acoustic modulation. Powder Technol 133(1):251-254

13. Qi L, Zeng X, Zhou J et al (2011) Stable micro-feeding of fine powders using a capillary with ultrasonic vibration. Powder Technol 214(2):237-242

14. Lu X, Yang S, Evans JRG (2008) Ultrasound-assisted microfeeding of fine powders. Particuology 6(1):2-8

15. Lu X, Yang S, Evans JR (2009) Microfeeding with different ultrasonic nozzle designs. Ultrasonics 49(6):514-521

16. Tardos GI, Lu Q (1996) Precision dosing of powders by vibratory and screw feeders: an experimental study. Adv Powder Technol 7(1):51-58

17. Matsusaka S, Urakawa M, Masuda H (1995) Micro-feeding of fine powders using a capillary tube with ultrasonic vibration. Adv Powder Technol 6(4):283-293

18. Crowder TM (2007) Precision powder metering utilizing fundamental powder flow characteristics. Powder Technol 173(3):217-223

19. Hickey AJ, Concessio NM (2010) Flow properties of selected pharmaceutical powders from a vibrating spatula. Part Part Syst Charact 11(6):457-462

20. Yang J, Chen R, Fan X et al (2006) Thermoelectric properties of silver-doped n-type $\mathrm{Bi}_{2} \mathrm{Te}_{3}$-based material prepared by mechanical alloying and subsequent hot pressing. J Alloys Compd 407(1):330-333

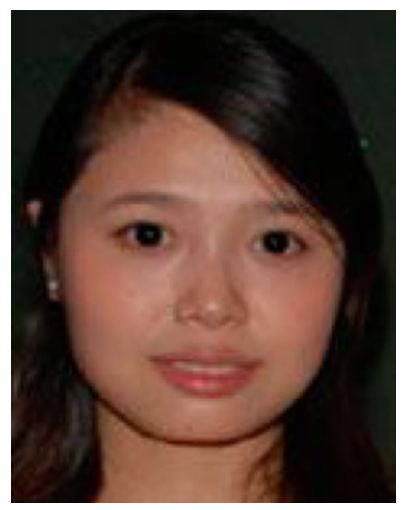

Hua-Yan Pu received her M. $\mathrm{Sc}$ and $\mathrm{PhD}$ degrees in Mechatronics Engineering from Huazhong University of Science and Technology (Wuhan, China), in 2007 and 2011, respectively. Since 2011, she has been a lecturer at Shanghai University (Shanghai, China). She was awarded the best paper in Biomimetics at 2013 IEEE International Conference on Robotics and Biomimetics. She was also nominated the best conference paper finalist at 2012 IEEE International Conference on Robotics and Biomimetics. Her current research interests include modeling, control, and simulation of field robotics and locomotion system.

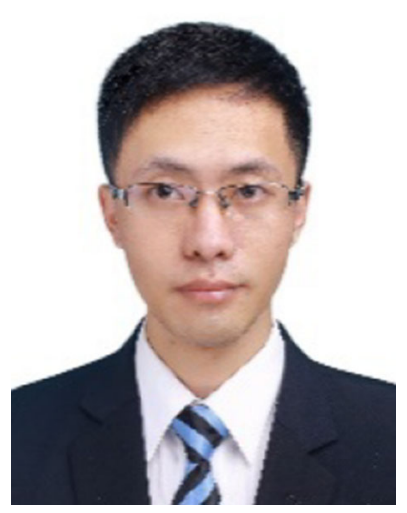

Rong-Qing Xie received his B.Eng. degree in Mechanical Engineering in 2016, and M. Eng. degree in Mechatronic Engineering in 2019, all from School of Mechatronic Engineering and Automation, Shanghai University (China). His research interests include vibration and noise reduction, and machine design.

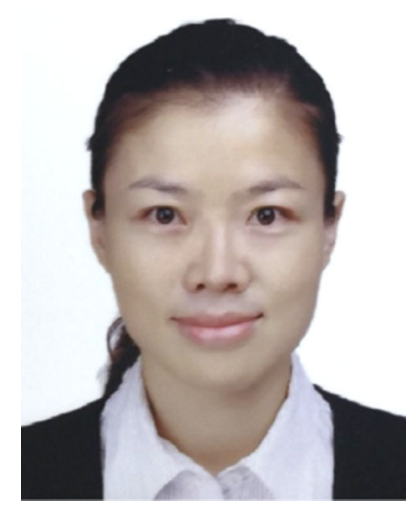

Yan Peng received her $\mathrm{PhD}$ degree in Pattern Recognition and Intelligent Systems from Shenyang Institute of Automation, Chinese Academy of Sciences, Shenyang, China, in 2009. Now, she is an associate professor at Shanghai University as executive dean of Research Institute of USV Engineering, Shanghai, China. Her current research interests include modeling and control of unmanned surface vehicles, field robotics and locomotion

system. 


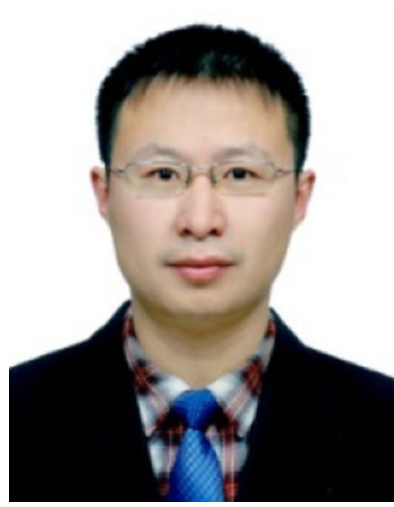

Yang Yang is a lecturer at School of Mechatronic Engineering and Automation, Shanghai University (Shanghai, China). He received his $\mathrm{PhD}$ degree in 2015 from Ritsumeikan University (Kyoto, Japan). From 2015 to 2016, he was a postdoctoral researcher at Department of Mechanical and Aerospace Engineering, Tokyo Institute of Technology (Tokyo, Japan). His research interests include soft robots, mobile robots, and terramechanics.

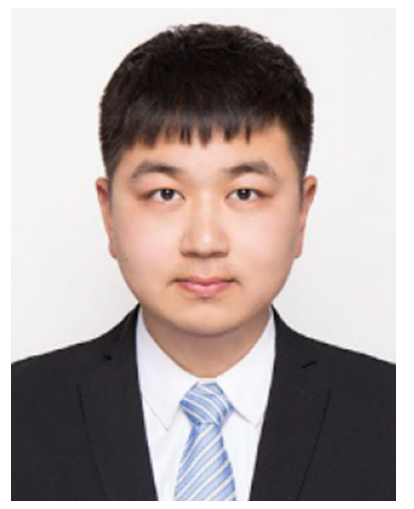

Shi-Yang He received his B.S. in Materials Science and Engineering from Zhengzhou University in 2017. He is a master candidate at school of materials science and engineering under the supervision of Prof. Jun Luo in Shanghai University. His research is focused on flexible thermoelectric materials and high throughput experiment to screen out the best performance of the thermoelectric material.

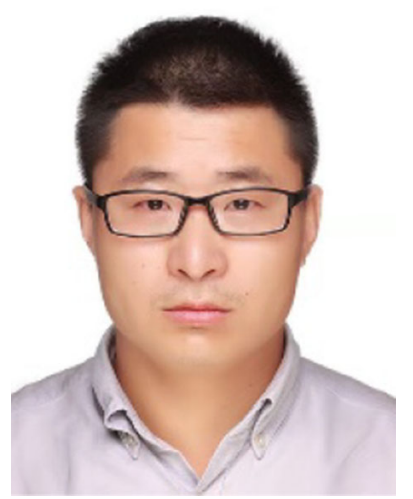

materials.

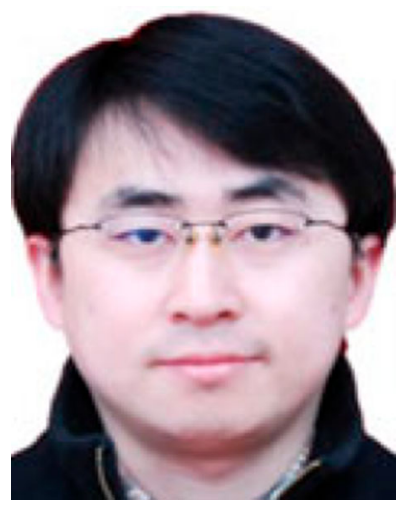

Yi Sun received his B. Eng. degrees in both Mechanical Engineering and in Computer Science and Technology in 2003, and M. Eng. degree in Mechatronic Engineering in 2006, all from Huazhong Univerisity of Science and Technology (Wuhan, China). In 2013, he received his Dr. Eng. degree in Robotics from Ritsumeikan University (Kyoto, Japan). From 2013 to 2016 he worked as a senior researcher at Ritsumeikan University. In July 2016, granted by the Program of Young Eastern Scholar of Shanghai, he joined Shanghai University as an Assistant Professor in School of Mechatronic Engineering and Automation. His research interests include bio-inspired robots, industrial robots and robotic vessels.

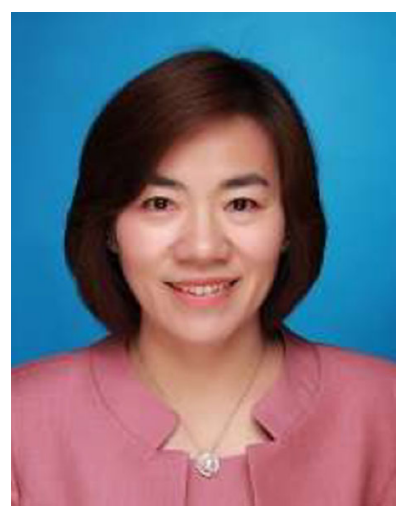

Shao-Rong Xie received the B. Eng. and M. Eng. degrees in mechanical engineering from Tianjin Polytechnic University, Tianjin, China, in 1995 and 1998, respectively, and the Dr. Eng. degree in mechanical engineering from the Institute of Intelligent Machines at Tianjin University and the Institute of Robotics and Automatic Information System, Nankai University, Tianjin, China, in 2001. She is a Professor with the School of Mechatronic Engineering and Automation, Shanghai University, Shanghai, China. Her research areas include advanced robotics technologies, bionic control mechanisms of eye movements, and image monitoring systems.

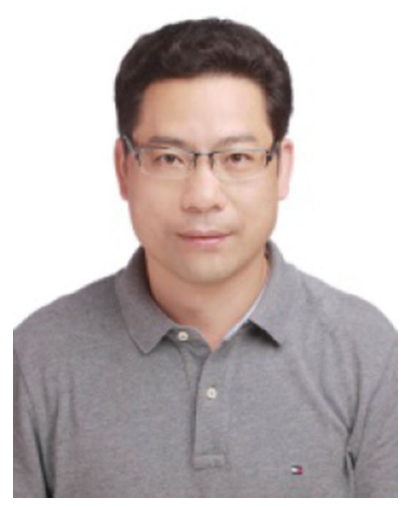

Jun Luo is a Professor in the School of Mechatronic Engineering and Automation of Shanghai University, China. He is the Head of Precision Mechanical Engineering Department in Shanghai University and the vice director of Shanghai Municipal Key Laboratory of Robotics. He received the B. Eng. degree in Mechanical Engineering from Henan Polytechnic University, Jiaozuo, China, in 1994, the M. Eng. degree in Mechanical Engineering from Henan Polytechnic University, Jiaozuo, China, in 1997, and the Dr. Eng. degree in Research Institute of Robotics in Shanghai Jiao Tong University, Shanghai, China, in 2000. His research areas include robot sensing, sensory feedback, mechatronics, man-machine interfaces, and special robotics. 